\title{
The use of Composite Pulses for improving DEER signal at $94 \mathrm{GHz}$
}

\author{
Claire L. Motion ${ }^{\mathrm{a}}$, Scott L. Cassidy ${ }^{\mathrm{a}}$, Paul A. S. Cruickshank ${ }^{\mathrm{a}}$, Robert I. \\ Hunter $^{\mathrm{a}}$, David R. Bolton ${ }^{\mathrm{a}}$, Hassane El Mkami ${ }^{\mathrm{a}}$, Sabine Van Doorslaer ${ }^{\mathrm{b}}$, \\ Janet E. Lovett ${ }^{\mathrm{a}}$, Graham M. Smith ${ }^{\mathrm{a}, *}$ \\ ${ }^{a}$ SUPA, School of Physics \& Astronomy, University of St Andrews, North Haugh, St \\ Andrews, Fife, KY16 9SS, United Kingdom \\ ${ }^{b}$ Department of Physics, University of Antwerp, Antwerp, 2610, Belgium.
}

\section{Abstract}

The sensitivity of pulsed electron paramagnetic resonance (EPR) measurements on broad-line paramagnetic centers is often limited by the available excitation bandwidth. One way to increase excitation bandwidth is through the use of chirp or composite pulses. However, performance can be limited by cavity or detection bandwidth, which in commercial systems is typically 100-200 MHz. Here we demonstrate in a $94 \mathrm{GHz}$ spectrometer, with >800 $\mathrm{MHz}$ system bandwidth, an increase in signal and modulation depth in a 4-pulse DEER experiment through use of composite rather than rectangular $\pi$ pulses. We show that this leads to an increase in sensitivity by a factor of 3 , in line with theoretical predictions, although gains are more limited in nitroxide-nitroxide DEER measurements.

Keywords:

Composite pulses, Sensitivity enhancement, Echo simulation, DEER,

\footnotetext{
* Corresponding author

Email address: gms@st-andrews.ac.uk (Graham M. Smith)
} 
PELDOR, Broadband non-resonant, W-band, Instrumentation

\section{Introduction}

For many pulsed magnetic-resonance experiments a large factor determining sensitivity is the proportion of spins that can be excited. The number of spins and the fidelity of the excitation profile can be improved by using modulated pulses that use phase or amplitude changes rather than simple rectangular pulses. These work to increase excitation bandwidth and/or compensate for errors such as applied field inhomogeneity. Such pulses include composite, adiabatic, chirp or more general optimal control sequences [1-5]. NMR has been using these methods for almost 40 years but their adoption in EPR has been slower due to the increased technical demands of working at higher electromagnetic frequencies. However, EPR will benefit from increased excitation bandwidth and the ever more complex sequences that can be applied with the increased fidelity of excitation offered. Recent advances have been made in the development and integration of arbitrary waveform generators (AWGs). These systems become more difficult to implement directly at higher frequencies as the performance of filters, amplifiers and IQ mixers becomes more critical. Conversely, higher frequencies can offer increased sensitivity: we have previously shown that a non-resonant widebandwidth spectrometer operating at W-band (94 GHz) offers 20-30 times improvement over the commercial X-band $(9.5 \mathrm{GHz})$ system [6]. We show here how fixed-amplitude phase-modulated composite pulses can be implemented at $94 \mathrm{GHz}$ to increase excitation bandwidth and to compensate for applied field inhomogeneity. 
Composite pulses are composed of several contiguous sub-pulses of varying phase and length that produce an excitation $\left(\pi / 2,90^{\circ}\right)$ or an inversion $\left(\pi, 180^{\circ}\right)$ of spin packets over a larger bandwidth than an equivalent standard rectangular pulse. Two of the advantages of composite pulses, over more complex sequences, is that they are still relatively short, typically three times the length of a rectangular inversion pulse, and that they can be used in existing spectrometers that have a phase-cycling capability.

Composite pulses have been used in NMR for a number of years to provide improvements in a range of areas. They were first demonstrated in EPR experiments by the Freed group in 1989, where an excitation bandwidth over $200 \mathrm{MHz}$ was demonstrated [7]. Morton et al. have also utilized the narrow bandwidth $\mathrm{BB} 1$ composite pulse sequence to improve $\mathrm{B}_{1}$ inhomogeneity for quantum computing applications [8]. Composite pulses have also been used by Turro et al. to enhance inversion recovery experiments on nitroxides at $\mathrm{X}$-band $[9]$.

One frequently used experiment that can benefit from composite pulses is the double electron electron resonance (DEER, also known as PELDOR) sequence, Figure 1, which is a pump-probe technique $[10,11]$. In the 4-pulse version the pump pulse inverts spins at one frequency and the probe uses a refocused echo sequence. In this way the dipole-dipole interaction can be measured and this can be related to distances in the 2 to, at least, $10 \mathrm{~nm}$ range [12-14].

To a first approximation the sensitivity of the DEER experiment is proportional to the fraction of spins excited by both the pump and probe pulses. This raises a significant challenge for broad-line systems such as metal ions 


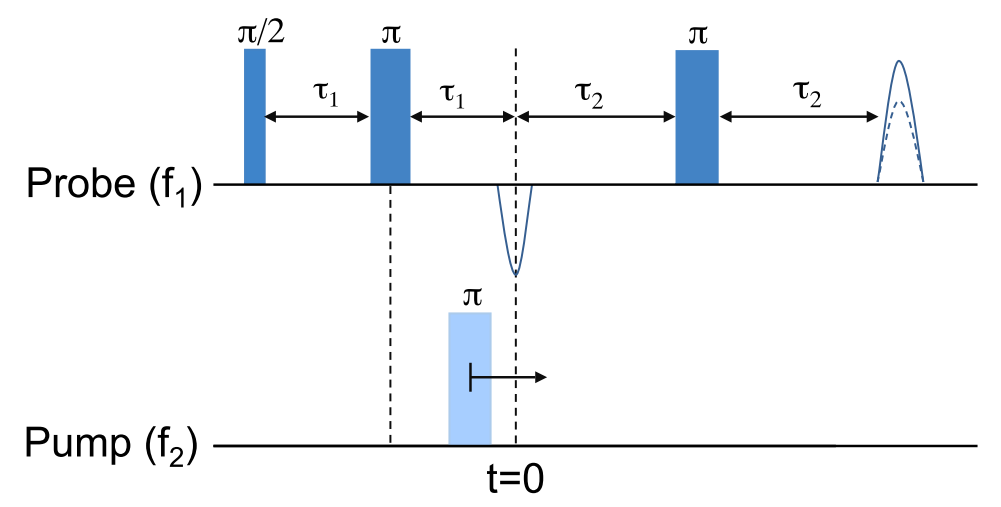

Figure 1: Standard 4-pulse DEER sequence, consisting of a refocused Hahn echo sequence on the probe or detection $\left(f_{1}\right)$ sequence, and a single inversion pulse on the pump spin $\left(f_{2}\right)$ sequence.

in metalloproteins which would otherwise potentially be very useful markers as intrinsic paramagnetic centers. Thus most applications of DEER in biomolecules have employed nitroxyl spin labelling, though there are notable exceptions [15-17]. Many of the modulated pulse techniques employed so far have focused on replacing the pump pulse where increases in modulation depth by up to a factor of 3 have been demonstrated [3, 5, 18]. Chirp and other modulated pulses have been utilized coherently to produce enhanced Hahn echoes for use in another dipole spectroscopy experiment, SIFTER (single frequency technique for refocusing dipolar couplings) [19-21].

Recently we reported that our $1 \mathrm{~kW}$ wideband $(>800 \mathrm{MHz}) \mathrm{W}$-band spectrometer, HiPER, can be used to measure the dipolar interaction, and therefore distance, between nitroxyl spin labels and a spin-half ferric (Fe(III)) heme in proteins [6]. We reported substantial increases in the signal-to-noise of the DEER measurement partly from improvements in both the pump and 
probe excitation bandwidth. This is despite the large $g$-anisotropy of the ferric-heme, which resulted in an absorption profile on the order of $1.5 \mathrm{~T}$ at W-band.

In this paper, we discuss the choice of composite pulse sequence to replace the $\pi$ pulses in the DEER experiment (Figure 1. We show the implementation on HiPER using a 4 channel 16-state vector modulator to provide phase control with 4 ns sub-pulse resolution. Finally, we demonstrate the gains of using composite pulses for echo and DEER sequences on extremely broad-line systems such as iron-heme and compare them to nitroxyl-nitroxyl systems.

\section{Composite Pulses}

\subsection{Background}

Composite pulses were first reported by Levitt et al. [1] demonstrating a simple sequence of 3 contiguous pulses that performed the same action as a $180^{\circ}$ pulse, but compensated for $B_{1}$ inhomogeneity across the sample and increased excitation bandwidth. Composite pulses are commonly described using the following notation [22]: $\left(\beta_{1}^{0}\right)_{\phi 1}\left(\beta_{1}^{0}\right)_{\phi 1} \ldots\left(\beta_{n}^{0}\right)_{\phi n}$ where $\left(\beta_{p}^{0}\right)$ describes the nominal flip angle (usually $90^{\circ}$ or $180^{\circ}$ ) of the sub-pulse p, and $\phi \mathrm{p}$ describes its phase, or axis of rotation. Levitt's original $180^{\circ}$ equivalent composite pulse, $90_{90} 180_{0} 90_{90}$ (when applied to a set of spins at equilibrium, $\left.\mathrm{M}_{z}\right)$ thus equates to a $90^{\circ}$ rotation around the $\mathrm{y}$-axis $\left(\phi=90^{\circ}\right)$, rotating the spins into the transverse plane along $\mathrm{x}$, followed by a $180^{\circ}$ rotation around this axis $\left(=0^{\circ}\right)$, finishing with another $90^{\circ}$ rotation around the y-axis. The sequence can compensate for $\mathrm{B}_{1}$ inhomogeneity by helping or hindering spins that under and over-rotate due to spatial applied field inhomogeneity. The 
same argument applies for compensation for frequency offset $\left(\Delta \omega_{1}\right)$ between the resonance frequency of the spin and that of the pulse frequency. This manifests itself as an increase in effective flip angle of the spins, which can be considered as the rotation axis being tilted by an angle $\Delta$ towards $+\mathrm{z}$.

\subsection{Inversion pulses}

In the absence of spin coupling, the net effect of various composite pulses can be easily calculated by applying appropriate rotation matrices, and visualized using contour plots that show the magnitude of the inversion as a function of frequency offset (x-axis) and $\mathrm{B}_{1}$ inhomogeneity (y-axis) [22], shown in Figure 2. $\Omega_{0}$ is the resonant frequency and is the frequency of the applied field/pulse, similarly, $\mathrm{B}_{1}^{0}$ is the nominal applied field amplitude to produce the required flip angle of the pulse (typically $\pi / 2$ or $\pi$ ). Figure 2 gives several examples for some of the most useful short wideband composite $\pi$-inversion pulses. In the contour/density plots, the magnitude of the inversion varies from $\mathrm{M}_{z}=+1$ (non-inverted) to $\mathrm{M}_{z}=-1$ (inverted).

Of the composite pulses shown in Figure 2, the $90_{0} 180_{180} 270_{0}$ sequence [23] offers the largest compensation for frequency offset, but only compensates for $\mathrm{B}_{1}$ at large frequency offsets, relative to the standard $\pi$ pulse. The 90 ${ }_{90} 180_{0} 90_{90}$ sequence [1] offers slightly less bandwidth but offers more $\mathrm{B}_{1}$ compensation at line center (zero offset). It is also a shorter sequence and only uses $90^{\circ}$ phase changes reducing potential distortion effects during the rapid phase changes. The bandwidth enhancement at nominal applied field $\left(\mathrm{B}_{1} / \mathrm{B}_{1}^{0}=1\right)$ for $90_{0} 180_{180} 270_{0}, 90_{90} 180_{0} 90_{90}$ and a $16 \mathrm{~ns} \pi$ rectangular $\left(180_{0}\right.$ in Figure 2) are shown in Figure 3(a).

On the other hand, the $90_{0} 360_{120} 90_{0}$ sequence is better at compensating 

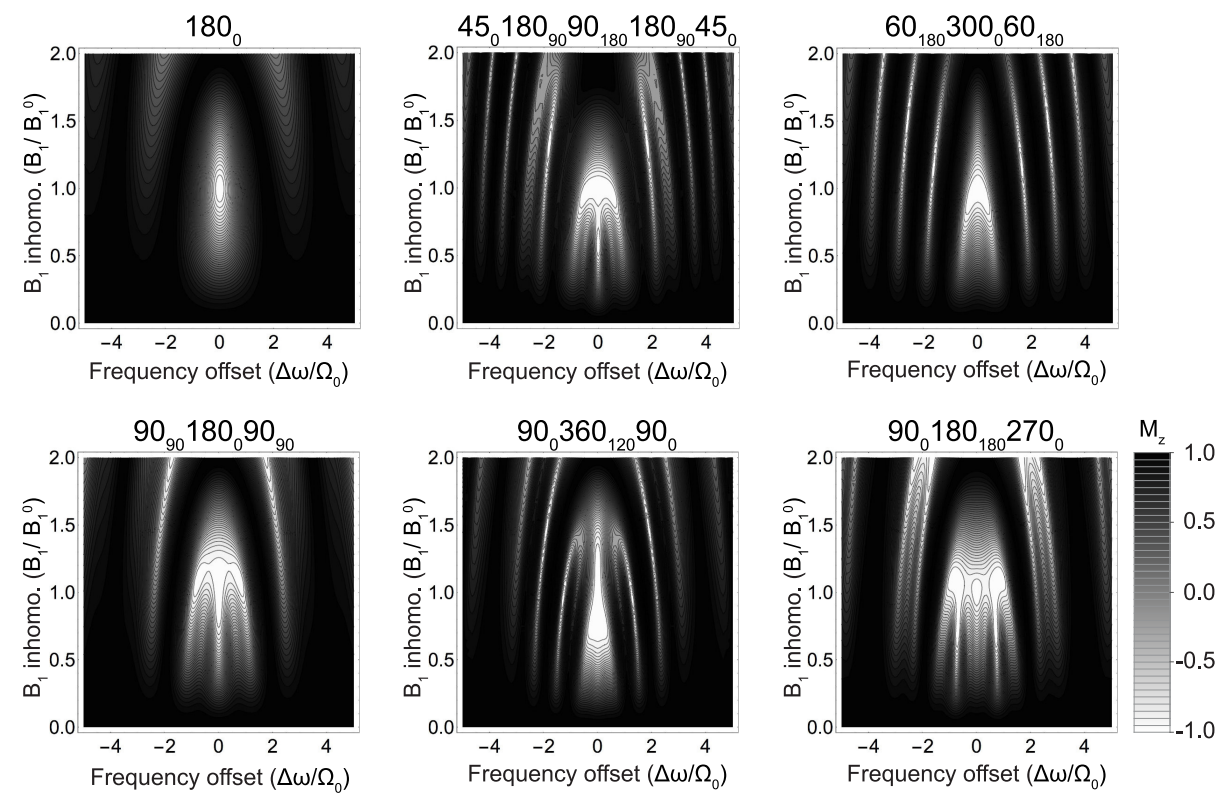

Figure 2: Contour plots showing the inversion performance of a range of composite pulses in terms of their ability to invert across frequency offset (x-axis) and in the presence of $\mathrm{B}_{1}$ inhomogeneity (y-axis), where $\Omega_{0}$ is the resonant frequency and $\mathrm{B}_{1}^{0}$ is the nominal amplitude of the applied pulse. The plot scales between white $=95-100 \%$ inversion $\left(\mathrm{M}_{z}\right.$ $=-1)$ to black $=0 \%$ inversion $\left(\mathrm{M}_{z}=+1\right)$.

for $\mathrm{B}_{1}$ inhomogeneity (whilst offering some compensation for frequency offset) [24]. In a coupled spin system, it is normally assumed that the $B_{1}$ field associated with the pulse is larger than the dipolar coupling. In cases where this is not true, the $45_{0} 180_{90} 90_{180} 180_{90} 45_{0}$ sequence potentially offers better inversion performance [25]. All the sequences above are examples of variable rotation sequences, which means that the $180^{\circ}$ rotation is defined for only one of the Euler angles and the other angles are left unconstrained. One consequence is that unwanted phase variations are introduced as a function of frequency offset. This effect is not important for an inversion pump pulse, 
but has the effect of introducing major distortions in a Hahn echo experiment [26]. However, these phase distortions can also be canceled by applying an identical second composite $\pi$-pulse, leading to an enhanced refocused echo.

The $60_{180} 300_{0} 60_{180}$ sequence [27] on the other hand is an example of a constant rotation pulse that can be used directly in Hahn echo experiments, but the bandwidth and $\mathrm{B}_{1}$ compensation are less than the variable rotation pulses for refocused echoes. This makes high performance variable rotation pulses suitable for 4-pulse DEER, but not 3-pulse DEER detection sequences.

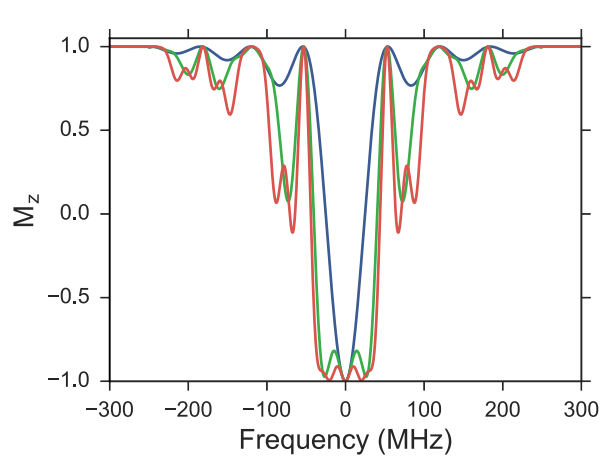

(a)

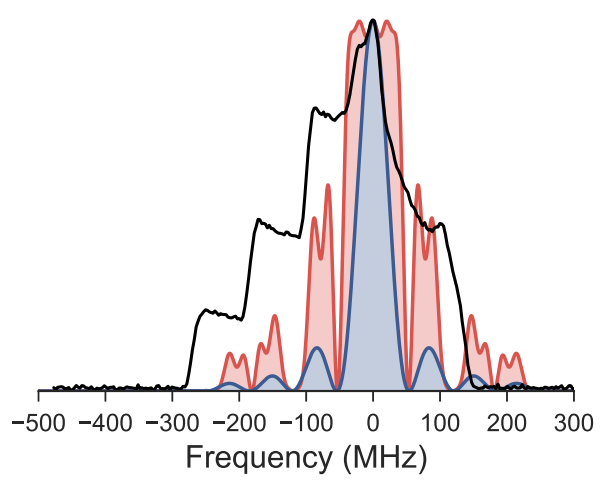

(b)

Figure 3: (a) Simulated inversion profile $\left(\mathrm{M}_{z}\right)$ of rectangular $\pi$ (blue), $90_{0} 180_{180} 270_{0}$ (red) and $90_{90} 180_{0} 90_{90}$ (green) composite pulses. The rectangular pulse is 16 ns $\pi$. (b) Simulated inversion profile of the $16 \mathrm{~ns}$ rectangular $\pi$ pulse (blue) and $90_{0} 180_{180} 270_{0}$ (red) composite pulse overlaid on the absorption spectra of a nitroxyl radical (black), taken at W-band. 


\section{Instrumentation}

\subsection{W-band spectrometer}

\subsubsection{General system overview (including non-resonant sample holder)}

All experiments were conducted on the home-built $1 \mathrm{~kW}$ pulsed $\mathrm{W}$-band spectrometer HiPER that has been described previously [28], where all of the pulse control is implemented at low frequencies and the $94 \mathrm{GHz}$ transmitted signal is generated via a x12 frequency multiplier as shown in Figure 5(a). This signal is then amplified to above $1 \mathrm{~kW}$ using an extended interaction klystron amplifier (EIKA) with a $1 \mathrm{GHz}$ bandwidth (Communications and Power Industries Canada, Inc. Georgetown, ON, Canada). This W-band instrument uses a non-resonant cavity and thus benefits from a very flat instrumental frequency response where there is less than $0.5 \mathrm{~dB}$ variation across a multi-GHz range, and the system bandwidth is dominated by the $800 \mathrm{MHz}(3 \mathrm{~dB})$ frequency response of the EIKA, shown in Figure 4.

HiPER utilizes a non-resonant induction-mode sample holder operating in reflection-mode. Samples are placed in holders consisting of $2.97 \mathrm{~mm}$ outerdiameter FEP (fluorinated ethylene propylene) tubes positioned within a 3 mm diameter circular waveguide supporting the $\mathrm{TE}_{11}$ mode. Power coupling from and to the sample may be adjusted by varying the position of a piezomounted back-short (mirror) situated below the sample (Attocube Systems AG, ANPz 101). Samples are typically multiple wavelengths long and of

similar volume to those used at X-band. This configuration results in a standing wave within the sample, and a relatively large 1 inhomogeneity, which is taken into account in the simulations. However, due to the large sample volumes used (typically 120-150 $\mu \mathrm{L}$ ), and the high power available at 


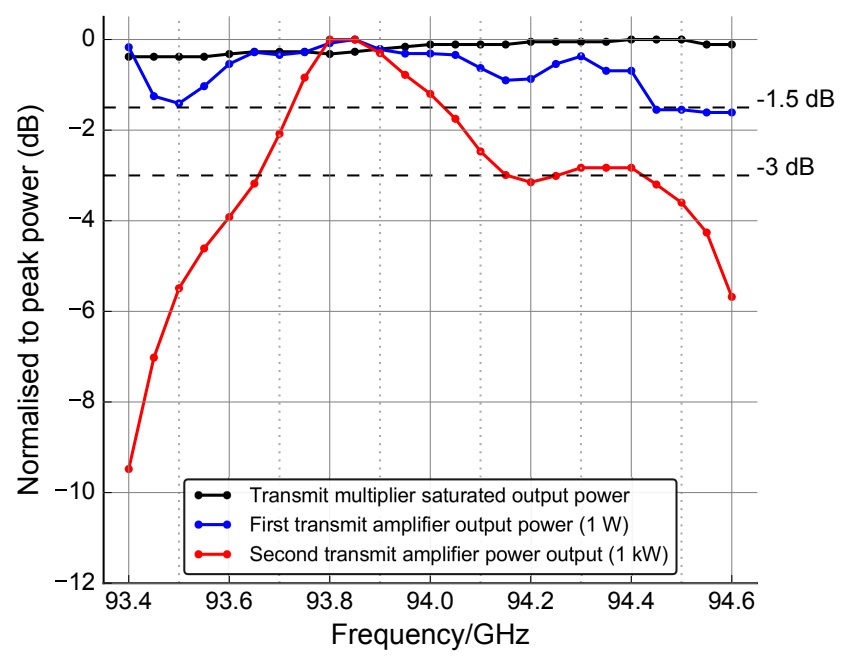

Figure 4: Power levels vs frequency at various stages in the transmit chain of the W-band spectrometer, normalized to the peak output. The response of the transmit multipliers (black) and the first transmit amplifier (1 W, blue) is relatively flat over the range 93.4$94.6 \mathrm{GHz}$, in comparison with the second transmit amplifier (1 kW EIKA, red) which causes the largest bandwidth limitation

this frequency, the concentration sensitivity can still be extremely high and is typically 20-30 times greater compared to the standard DEER measurements at X-band with nitroxides [29]. Further details of the setup can be found in reference [28].

\subsubsection{Annealing}

In addition to optimization of the position of the back-short/mirror for power coupling into the sample, it has also been found that the use of FEP tubes and an annealing step has often significantly improved the observed echo signal in low temperature measurements on HiPER. This increase in 
signal is attributed to the formation of a better glass and reduction of the effects of dielectric scattering within the sample. The formation of better glasses has also been visually observed after rapid sample freezing with liquid nitrogen when using FEP tubes relative to quartz tubes. This effect is attributed to less mechanical stress being placed in the sample during freezing due to the hydrophobic interface of the FEP tube. Quartz tubes, on the other hand, are more hydrophilic and have a very low coefficient of expansion relative to most samples. More consistent results (especially with respect to quartz tubes) have also been obtained by annealing the sample after coldloading into the spectrometer at $130 \mathrm{~K}$. In this step the temperature inside the cryostat is increased to the glass transition temperature, (where the microwave loss of the sample is observed to increase rapidly). For a sample that uses a glycerol cryoprotectant of around 50\% glycerol, the glass transition is expected to occur at approximately $170 \mathrm{~K}[30]$ and changes in the reflected microwave signals are generally observed in the 170 - $200 \mathrm{~K}$ range. At this point, the cryostat temperature is then lowered to 50 - $60 \mathrm{~K}$ or to the desired target measurement temperature. The benefits of this annealing step are attributed simply to the release of stress within the sample allowing lower temperatures to subsequently be reached without sample cracking.

\subsubsection{Phase modulator}

The original phase control box used in the HiPER spectrometer [28] (shown schematically in Figure 5(a)) was replaced by constructing a new 4-channel, 16-state vector modulator as shown in Figure 5(b). The 7.833 $\mathrm{GHz}$ input signal is applied to a $0^{\circ} 2$-way power splitter. One arm acts as a phase reference, while the other arm is split into four channels, each contain- 
ing separate phase shifter/attenuator combinations that can be individually or collectively selected via fast switches controlled by a ParBERT pulse controller (Agilent, Inc.), which provides 4 ns timing resolution. The signals from these channels are subsequently recombined in phase quadrature with the signal from the reference arm. Thus, by varying the attenuation in a given phase channel, a continually variable phase shift (of up to $74^{\circ}$ ) can be obtained. However, because the signal is subsequently multiplied by 12 to the final transmission frequency, at $7.833 \mathrm{GHz}$ a maximum phase shift of only $30^{\circ}$ is required in order to produce a phase change of $360^{\circ}$ at $94 \mathrm{GHz}$. If, for example, we require 15 binary weighted steps over a 360 range at 94 $\mathrm{GHz}$, we require phase step intervals of $1.875^{\circ}, 3.75^{\circ}, 7.5^{\circ}$ and $15^{\circ}$ at 7.833 $\mathrm{GHz}$ from the 4 channels, which is easily attainable. Since the phase change is relatively small, the accompanying amplitude change is also very small and this is largely eliminated by the final leveling amplifier and the subsequent amplitude-leveling multiplication process to $94 \mathrm{GHz}$. Similar phase switching at $7.833 \mathrm{GHz}$ could be achieved with more flexibility with an Arbitrary Waveform Generator, although the filtering requirements become more stringent at high frequencies pre-multiplication. 


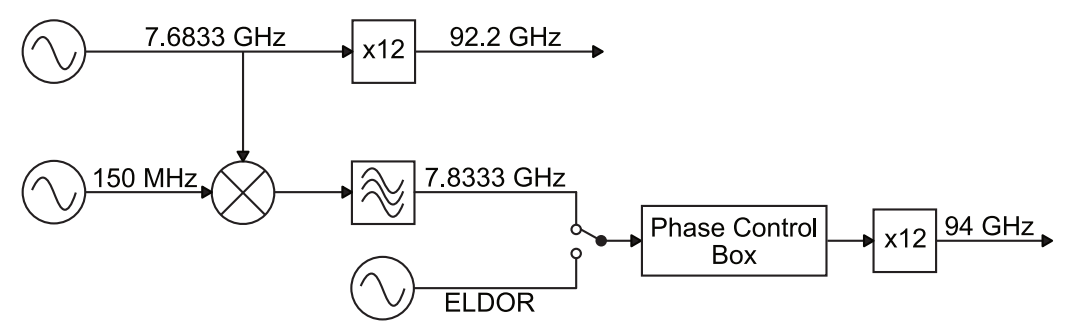

(a)

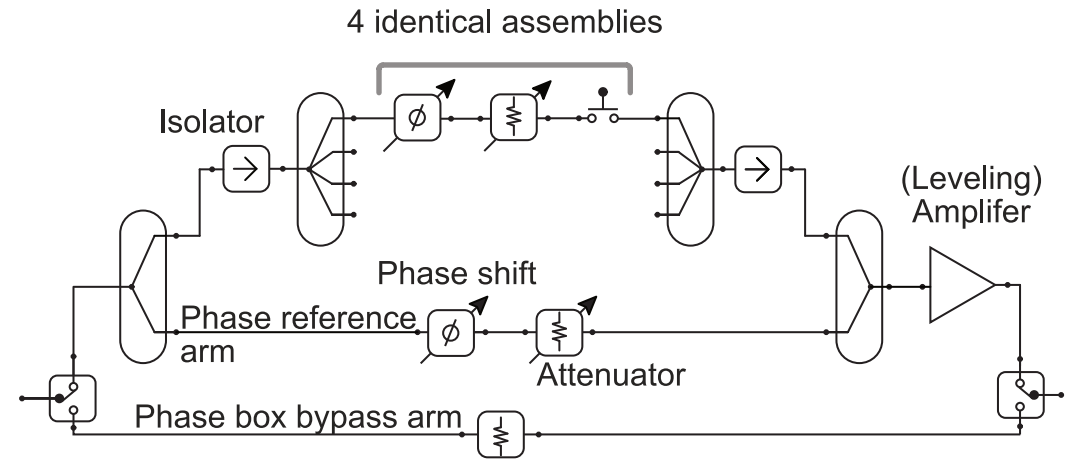

(b)

Figure 5: (a) Schematic diagram of the simplified frequency generation scheme used on HiPER, and the location of the phase control in the system. (b) Schematic diagram of the new phase box assembly, showing the implementation of 4 independent phase channels each consisting of a phase shifter, attenuator and channel selection switch. The scheme illustrates how the 4 channels can be combined as required by simultaneously opening several channel selection switches. It also shows the phase box bypass channel. 


\section{Materials and methods}

\subsection{Materials}

\subsubsection{Neuroglobin NGB-C120R1}

To demonstrate the effect of composite pulses on broadband samples for refocused echo enhancement, a low-spin Fe(III) spin-labelled human neuroglobin, NGB was used. The mutant NGB-C120R1 used here was labeled with MTSL (1-oxyl-2,2,5,5-tetramethylpyrroline-3-methyl) at the cysteine site, 120. This site is on the G-helix of the neuroglobin, and has an approximate separation between the Fe(III) and label of $2.1 \mathrm{~nm}$. It was prepared as given in [31].

NGB-C120R1 protein solutions ( $\mathrm{pH} 8.5)$ were mixed 1:1 with glycerol leading to final concentration of $1.1 \mathrm{mM}$ (heme content) and then flash frozen in FEP sample tubes before cold loading into the spectrometer at $140 \mathrm{~K}$. The samples were then annealed by gradual temperature raising to $180 \mathrm{~K}$ for a short time, before recooling/glassing the sample to the measurement temperature of $6 \mathrm{~K}$.

\subsubsection{Model nitroxyl biradical MSA236}

The rigid nitroxyl biradical MSA236 (compound IIIb in [32]) was used for composite refocused echo and DEER comparison experiments due to its well defined distance $(4.1 \mathrm{~nm})$ minimizing any potential errors due to instantaneous diffusion. Its structure is shown in Figure 6. The biradical was set in deuterated ortho-terphenyl (d8-oTP) with biradical concentration of $50 \mu \mathrm{M}$ and was loaded into a FEP tube before melting the sample inside the tube with a heat gun at approximately $80^{\circ} \mathrm{C}$. The sample was then allowed to re- 
crystallize at ambient (room) temperature before testing in the spectrometer at room temperature.

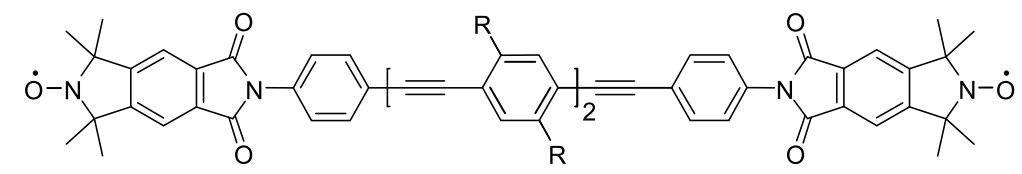

Figure 6: Structure of MSA236. The R groups are C6H13.

\subsection{Methods}

\subsubsection{Pulsed Experiments}

All refocused echo experiments on the low spin Fe(III) neuroglobin were carried out using a continuous flow helium cryostat (CF935) and a temperature control system (ITC 502) from Oxford Instruments. This was carried out at $6 \mathrm{~K}$ using the observer part of the DEER sequence (Figure 1), $\pi / 2$ $\tau_{1}-\pi-\left(\tau_{1}+\tau_{2}\right)-\pi-\tau_{2^{-}}$echo with pulse lengths $\mathrm{t}_{\pi / 2}=8 \mathrm{~ns}$ and $\mathrm{t}_{\pi}=16 \mathrm{~ns}$ for standard pulses and $\mathrm{t}_{\pi \text { composite } 1}=8+16+8 \mathrm{~ns}\left(90_{90} 180_{0} 90_{90}\right.$, Levitt [1]) or

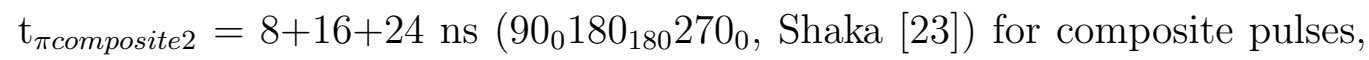
with inter-pulse delays of $\tau_{1}=198 \mathrm{~ns}$ and $\tau_{2}=954 \mathrm{~ns}$.

Refocused echo experiments on MSA236 were carried out at room temperature $(291 \mathrm{~K})$ using the same sequence and pulse lengths as for the neuroglobin, with inter-pulse delays of $\tau_{1}=250 \mathrm{~ns}$ and $\tau_{2}=750 \mathrm{~ns}$.

DEER experiments were carried out on MSA236 at room temperature $(291 \mathrm{~K})$ using the four-pulse DEER sequence (Figure 1$), \pi / 2\left(\mathrm{f}_{\text {probe }}\right)-\tau_{1}-\pi\left(\mathrm{f}_{\text {probe }}\right)$ - $\left.\left(\tau_{1}+\mathrm{t}\right)-\pi\left(\mathrm{f}_{\text {pump }}\right)-\tau_{2}-\mathrm{t}\right)-\pi\left(\mathrm{f}_{\text {probe }}\right)-\tau_{2}-e c h o$, with the same pulse lengths as the refocused echo experiment and inter-pulse delays of $\tau_{1}=250 \mathrm{~ns}$ and $\tau_{2}=$ 2450 ns. The observer pulse frequency was $93.79944 \mathrm{GHz}$ and the pump was 
94.029 GHz, providing an offset of $230 \mathrm{MHz}$. The pump pulse started $100 \mathrm{~ns}$ after the first $\pi$-pulse on the probe sequence, and was incremented in 8 ns steps. The sequence repetition frequency was $30 \mathrm{kHz}$, averaging 30,000 shots per point.

The field swept echo experiment on MSA236 was carried out at $291 \mathrm{~K}$ using a Hahn echo sequence, $\pi / 2-\tau-\pi-\tau-e c h o$, with 7 and 14 ns $\pi / 2$ and $\pi$ pulse lengths and inter-pulse delay $\tau=250 \mathrm{~ns}$. The field was swept from $3337 \mathrm{mT}$ to $3367 \mathrm{mT}$ in $0.1 \mathrm{mT}$ steps, with pulse frequency of $93.9996 \mathrm{GHz}$. Sequence repetition frequency was $5 \mathrm{kHz}$, averaging 5,000 shots per point.

\section{Results}

\subsection{Simulation of excitation profiles for composite pulse sequences}

\subsubsection{Hahn echo and refocused Hahn echoes}

The effects of constant and variable phase rotation composite pulses in echo sequences has been modeled in Mathematica. An arbitrary $\mathrm{B}_{1}$ amplitude profile across the sample can be specified and an arbitrary lineshape can also be specified. For HiPERs non-resonant sample holders, operating in induction mode, it is assumed the sample is lossless, and the $\mathrm{B}_{1}$ profile along the length of the sample is sinusoidal and its radial distribution can be approximated by a $\mathrm{TE}_{11}$ mode in a dielectrically loaded circular waveguide $[33,34]$, taking into account the $0.5 \mathrm{~mm}$ thickness of the sample tube. This is given in cylindrical coordinates by the expression,

$$
B_{1}(r, \phi, z)=B_{1}^{\max }\left(J_{0}\left(1.84118 \frac{r}{a}\right)-J_{2}\left(1.84118 \frac{r}{a}\right) \cos (2 \phi)\right) \cos \left(\frac{\pi z}{2}\right)
$$


where $\mathrm{B}_{1}^{\max }$ is the maximum $\mathrm{B}_{1}$ amplitude present in the sample, $\mathrm{z}$ is along the length of the sample, $r$ is the radius of the sample, $a$ is the radius of the sample holder/cylindrical waveguide, and $\mathrm{J}_{0}$ and $\mathrm{J}_{2}$ are zeroth and second order Bessel functions of the first kind respectively. The factor of 1.84118 is derived from the boundary conditions of the waveguide for this mode. This results in a $B_{1}$ amplitude distribution, which has repeating symmetry over $\mathrm{r}=0 \rightarrow \mathrm{a}, \phi=0 \rightarrow \pi / 2$ and $\mathrm{z}=0 \rightarrow 1$, where the radius is scaled to take into account the different dielectric constants of the sample tube and the sample. The dielectric constant of the FEP tube was taken as $\epsilon_{F E P}=2.1$ at $60 \mathrm{~K}$ at $94 \mathrm{GHz}$. The sample, which typically consists of 50/50 glycerol/water content is taken to have a dielectric constant $\epsilon_{g / w}=3.5$ at $94 \mathrm{GHz}$ at $60 \mathrm{~K}$, similar to that measured in Reference [35]. This allows the $\mathrm{B}_{1}$ density profile over the volume to be calculated. Further details about $\mathrm{B}_{1}$ amplitude and density profile can be found in the SI.

The code then generates a 2-dimensional array that represent separate spin packets that each see different $B_{1}$ amplitudes and frequency offsets. This creates a distribution of $\mathrm{B}_{1}$ amplitudes within the sample ranging from $0 \leq \mathrm{B}_{1}^{0}$ $\leq \mathrm{B}_{1}^{\max }$, where $\mathrm{B}_{1}^{0}$ is the amplitude that produces the desired flip angle for a given pulse length at zerop frequency offset. The weighting of a spin packet at a particular $\mathrm{B}_{1}$ field is proportional to the volume of sample experiencing that field. Each spin packet at a given $\mathrm{B}_{1}$ amplitude and frequency offset is then acted upon by the appropriate rotation matrix for the relevant type of pulse, allowing for any composite pulse sequence to specified. The rotation matrices are defined by the flip angle, 


$$
\beta=\beta^{\prime} \sqrt{\left(\frac{B_{1}}{B_{1}^{0}}\right)^{2}+\left(\frac{\Delta \omega_{1}}{\Omega_{1}^{0}}\right)^{2}}
$$

and its axis of rotation,

$$
\vec{r}=\frac{B_{1}}{B_{1}^{0}}(\cos \phi \hat{i}+\sin \phi \hat{j})+\frac{\Delta \omega_{1}}{\Omega_{1}^{0}} \hat{k}
$$

where $\beta^{\prime}$ is the nominal flip angle at zero frequency offset (usually $90^{\circ}$ or $\left.180^{\circ}\right), \Omega_{1}^{0}$ is the resonant frequency of the spin, $\Delta \omega_{1}$ is the resonance frequency offset, $\phi$ is the phase and $\hat{i}, \hat{j}$ and $\hat{k}$ are unit vectors in the $\mathrm{x}, \mathrm{y}$ and $\mathrm{z}$ directions respectively.

Any period of free precession is defined by a delay matrix that rotates each of the vectors around the z-axis in the rotating frame by angle $\theta=\tau_{1} \Delta \omega_{1}$. The model then returns the sum of all the magnetization vectors around the time $2 \tau_{1}$ after the first pulse, showing the echo that is produced.

As an example, the resulting Hahn echo is shown in Figure 7(a) where the refocusing $\pi$-pulse has been replaced by a number of different composite pulses. The model assumes excitation of a broad line, which is flat over the range $-10 \leq\left(\Delta \omega_{1} / \Omega_{1}^{0}\right) \leq 10$, nominally given by the function, $\exp \left(-\left(\left(\Delta \omega_{1} / \Omega_{1}^{0}\right) / 9\right)^{16}\right)$. Further discussion regarding optimization of the $\mathrm{B}_{1}$ amplitude can be found in the Supporting Information. The plot shows distorted echoes for the variable rotation sequences, $90_{90} 180_{0} 90_{90}$ and $90_{0} 180_{180} 270_{0}$ sequences, as expected, and a slightly enhanced echo for the constant rotation composite pulse, $60_{180} 300_{0} 60_{180}$ in comparison with the Hahn echo produced with a rectangular $\pi$-pulse. 


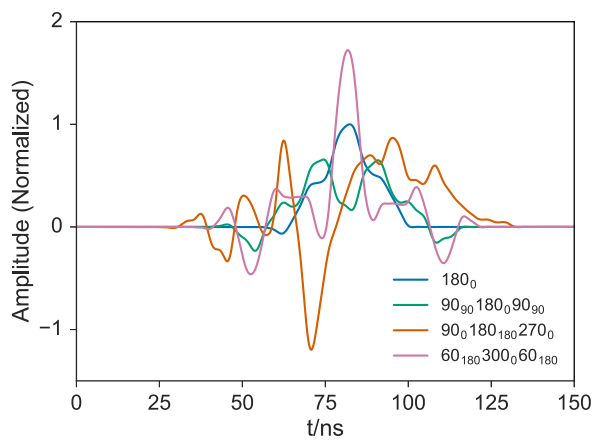

(a)

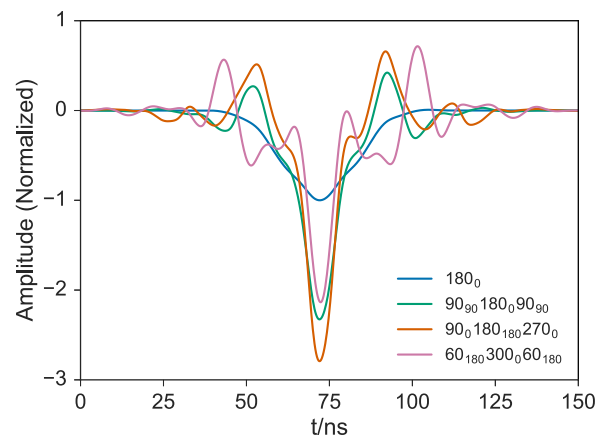

(b)

Figure 7: Simulations of (a) Hahn echoes and (b) refocused echoes using composite and rectangular $\pi$ pulses.

\subsubsection{Refocused Hahn echo}

The refocused Hahn echo was simulated by adding a second inversion pulse that matches the first inversion pulse, introduced a period $\tau_{2}$ after the echo. This produces an echo at $2\left(\tau_{1}+\tau_{2}\right)$ after the first pulse. In the composite refocused echo case, the phase distortion is reversed, producing an enhanced refocused echo, as shown in Figure 7(b). It indicates that of the short composite pulses shown, the $90_{0} 180_{180} 270_{0}$ sequence (orange) provides the largest enhancement in echo amplitude for partial excitation of broadline spectra, with echo amplitude enhancement of 2.79 times that provided by the rectangular pulse (blue).

\subsubsection{Refocused Hahn echo: homogeneous $B_{1}$ field}

The refocused Hahn echo simulation was repeated with a homogeneous $\mathrm{B}_{1}$ field, to reflect the conditions of standard cavity set ups as found in most commercial EPR systems. The $\mathrm{B}_{1}$ field in this case equals $\mathrm{B}_{1}^{0}$ over the sample. 
Figure 8 shows the resulting refocused echoes.

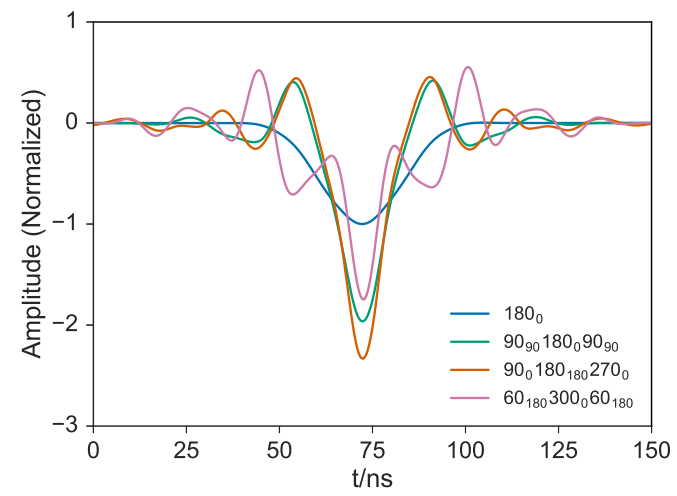

Figure 8: Simulation of refocused Hahn echoes using composite and rectangular $\pi$ pulses on a broad line with homogeneous $\mathrm{B}_{1}$ profile

The simulation indicates that composite pulses, in the absence of $\mathrm{B}_{1}$ inhomogeneity, can 9provide up to 2.33 times enhancement in echo amplitude using $90_{0} 180_{180} 270_{0}$ sequence when exciting broad line samples. When exciting narrow-line samples where standard pulses can already excite the majority of the line, the enhancement is negligible. It should be noted that effects of cavity bandwidth is not taken into account in this simulation.

\subsection{Time and frequency response of HiPER}

In any EPR system using high $\mathrm{B}_{1}$ and/or broadband pulses, it is important to determine the time and frequency response of the system, including the bandwidth limitations associated with high power amplification, cavity resonators and the detection system. In this system the frequency response is dominated by the $800 \mathrm{MHz}$ bandwidth associated with the high power $94 \mathrm{GHz}$ amplifier and the $1 \mathrm{GHz}$ intermediate frequency (IF) filter on the detection system. The frequency response of the rest of the front end of the 
spectrometer is relatively flat $(0.5 \mathrm{~dB}$ over several $\mathrm{GHz})$ due to the use of non-resonant sample holders and the extremely high isolation between source and sample, and sample and detector [28].

A typical $94 \mathrm{GHz}$ amplitude response for a $90_{0} 180_{180} 270_{0}$ composite pulse sequence, using a $16 \mathrm{~ns} \pi$ pulse length ( $\mathrm{t}_{\pi \text { composite } 2}=8+16+24=48 \mathrm{~ns}$ ), after multiplication (red) and after amplification by the $1 \mathrm{~kW}$ amplifier (blue), is shown in Figure 9(a). Both measurements were made using a fast planar

detector (Farran Technology, WDP-10) and a 20 GS/s digital oscilloscope (LeCroy) with $4 \mathrm{GHz}$ analog input bandwidth. The power output of the phase modulated $94 \mathrm{GHz}$ input signal, post multiplication, pre-amplification (red) is relatively clean, whereas the post amplification, high power signal (blue) shows ringing at the points where there is a rapid phase change, due to the $800 \mathrm{MHz}$ bandwidth of the amplifier. It should be noted that some of the distortion shown in Figure 9(a) is caused by cable bounce in the measurement system: for example, the spike seen at the end of the main pulse (blue, asterisk) is caused by this effect. Figure 9(b) shows the full response of the system after down-conversion using the IQ detection system built into the spectrometer. It should be noted in a conventional cavity-based system that the phase response would be expected to be much slower and more distorted and determined by the ring-down of the cavity.

\subsection{Simulations vs. experiment for composite pulse refocused echoes}

\subsubsection{Fe(III) neuroglobin}

Compared to the nitroxyl center, low-spin ferric heme centers exhibit a very large $g$-anisotropy with corresponding broad EPR spectra [6] and short relaxation times. Hence, pulsed EPR experiments on NGB-C120R1 need to 


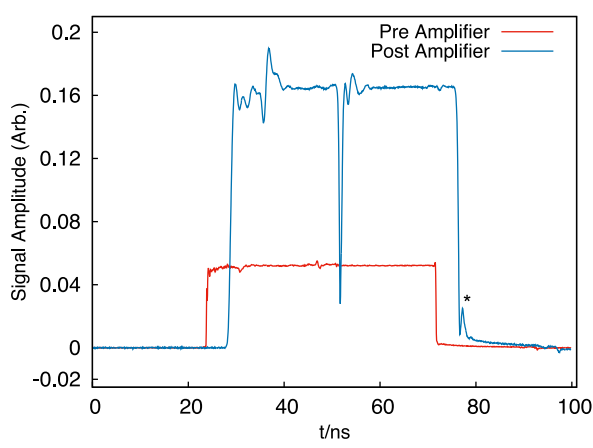

(a)

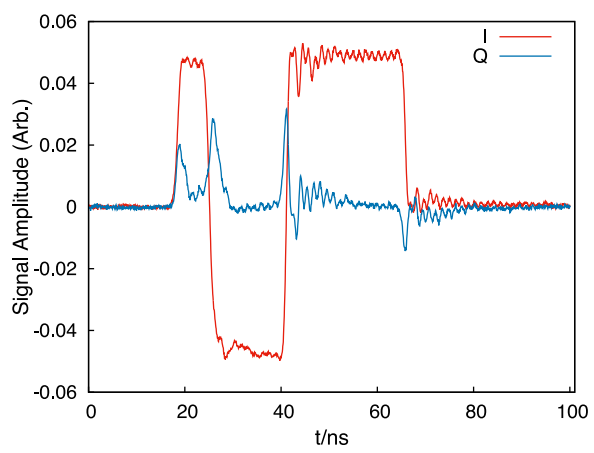

(b)

Figure 9: (a) Plots showing the pulse power profile of a $90_{0} 180_{180} 270_{0}$ composite pulse using $\left(\mathrm{t}_{\pi \text { composite } 2}=8+16+24=48 \mathrm{~ns}\right.$ pulse length, prior to (red) and after (blue) the 1 $\mathrm{kW}$ amplifier (blue), measured using a fast digital oscilloscope with $4 \mathrm{GHz}$ analog bandwidth. It shows minimal distortion during phase changes prior to the $1 \mathrm{~kW}$ amplifier, and ringing that resolves within a few ns after the $1 \mathrm{~kW}$ amplifier. (b) Output from the IQ detection system via the sample-holder showing the full system response.

be performed at very low temperatures.

Refocused echo measurements were made at $6 \mathrm{~K}$ where the short $\mathrm{T}_{1}$ of the $\mathrm{Fe}(\mathrm{III})$ center still permitted repetition rates of $1 \mathrm{kHz}$. In order to compare the experimentally obtained refocused spin echoes as recorded on the detection part of a DEER experiment, simulations of the refocused echo were carried out using the same approach as detailed in Section 5.1.2. Similarly to the echoes shown in Figure 7, the EPR spectrum of the Fe(III) center was taken as flat over the excitation profile, and the model took into account the $\mathrm{B}_{1}$ inhomogeneity profile of the non-resonant sample holder in HiPER. Figure 10 shows excellent agreement between both experimentally measured spin echo of the $\mathrm{Fe}(\mathrm{III})$ center at $6 \mathrm{~K}$ and simulated refocused echo, for both rectangu- 
lar $\pi$-pulses and $90_{0} 180_{180} 270_{0}$ composite $\pi$-pulses. The $90_{0} 180_{180} 270_{0}$ was chosen here for comparison as the simulation indicated it should provide the largest enhancement in terms of echo amplitude and the largest bandwidth of excitation. Echo amplitudes of composite pulses in both experiment and simulation have been normalized to the standard pulse amplitude. The small bump seen to the right of the echo peaks in both experiment and simulation (indicated by an asterisk) is a consequence of the high $\mathrm{B}_{1}$ inhomogeneity across the sample at W-band.

The simulation indicated that 2.79 times enhancement in echo height be theoretically expected, whereas the experiment shows a 2.73 enhancement. In a DEER experiment the more important quantity is the integrated echo signal-to-noise ratio. In both cases an increase of 1.8 is observed, assuming rectangular integration windows whose width is defined by the $30 \%$ echo height points, as indicated in Figure 10(b, hatched). This excellent agreement with theory is achieved for the special case where the line shape is essentially flat over the excitation profile and where the effects of instantaneous diffusion are expected to be negligible. It also shows that the excitation is not limited by the system bandwidth. 


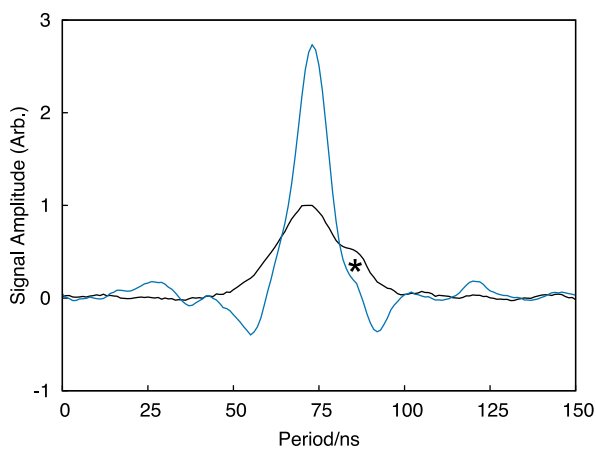

(a)

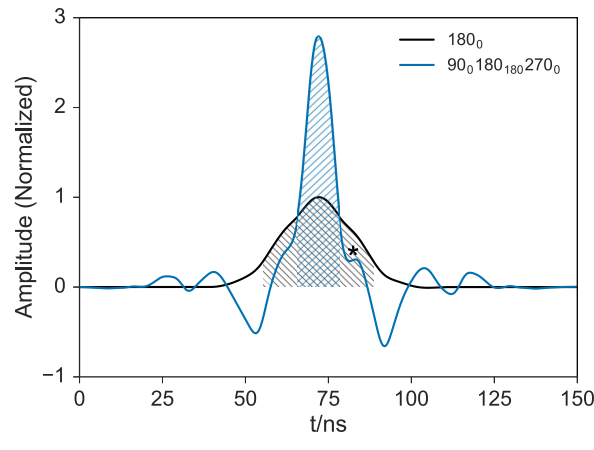

(b)

Figure 10: (a) Experimentally measured refocused spin echoes of a frozen solution of NGB-C120R1 obtained using the $90_{0} 180_{180} 270_{0}$ composite $\pi$-pulse (blue) and standard rectangular $\pi$-pulse (black). (b) Simulation of the refocused spin echo, using the same sequences, assuming a flat linewidth over the excitation profile and taking into account the large $B_{1}$ inhomogeneity associated with HiPER. The asterisk indicates the bump caused by the large $\mathrm{B}_{1}$ inhomogeneity of HiPER (see text).

\subsubsection{Nitroxyl biradical refocused echoes}

A more general application is the one where a nitroxide biradical is used. However, here the profile is not flat (over the excitation range) and the effects of both inter and, intra-molecular instantaneous diffusion, and excitation of forbidden transitions (in the $\mathrm{X}$ and $\mathrm{Y}$ parts of the spectrum), are not necessarily negligible. Composite refocused echoes were measured on the MSA236 model nitroxyl biradical system at different field positions/orientations on the EPR spectrum nitroxyl center. Echoes were obtained using standard, rectangular $\pi / 2$ and $\pi$ pulses and with the equivalent $90_{90} 180_{0} 90_{90}$ and $90_{0} 180_{180} 270_{0}$ composite $\pi$-inversion pulses. In each case, the initial $\pi / 2$ excitation pulse was provided by an $8 \mathrm{~ns} \pi / 2$ rectangular pulse. The field 
positions used as shown in Figure 11(a) are $\mathrm{X}=3347 \mathrm{mT}, \mathrm{Y}=3350 \mathrm{mT}$, $\mathrm{Z}_{1}=3352 \mathrm{mT}, \mathrm{Z}_{2}=3355 \mathrm{mT}$ and $\mathrm{Z}_{3}=3358 \mathrm{mT}$. This field swept echo spectrum of the biradical (using rectangular pulses) also shows the effects of instantaneous diffusion where the $\mathrm{X}$ and $\mathrm{Y}$ components are attenuated with respect to the Z-components. The results of the refocused echo experiments for each of the composite and standard sequences versus field positions are shown in Figure 11(b). The enhancements provided are summarized in Table 1.

\begin{tabular}{|l|l|l|}
\hline Orientation & $\begin{array}{l}\text { Normal vs. } \\
\mathbf{9 0}_{90} \mathbf{1 8 0}_{0} \mathbf{9 0}_{90} \\
\text { Composite }\end{array}$ & $\begin{array}{l}\mathbf{9 0}_{0} \mathbf{1 8 0}_{180} \mathbf{2 7 0}_{0} \\
\text { Composite }\end{array}$ \\
\hline $\mathbf{X}$ & 1.266 & 1.217 \\
\hline $\mathbf{Y}$ & 1.138 & 1.063 \\
\hline $\mathbf{Z}_{1}$ & 1.219 & 1.197 \\
\hline $\mathbf{Z}_{2}$ & 1.406 & 1.462 \\
\hline $\mathbf{Z}_{3}$ & 1.621 & 1.783 \\
\hline
\end{tabular}

Table 1: Echo enhancement with field position for composite versus rectangular refocused echoes, normalized to the rectangular echo amplitude 


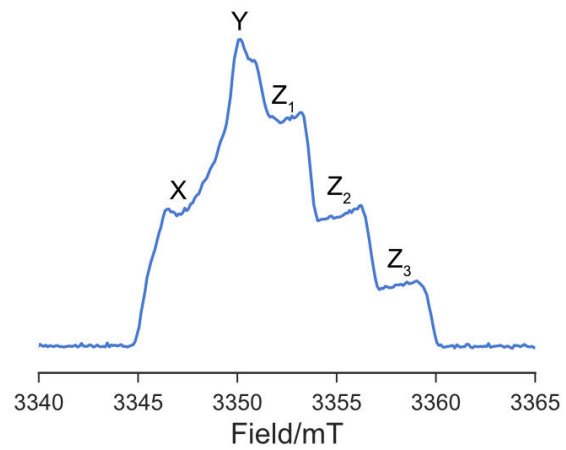

(a)

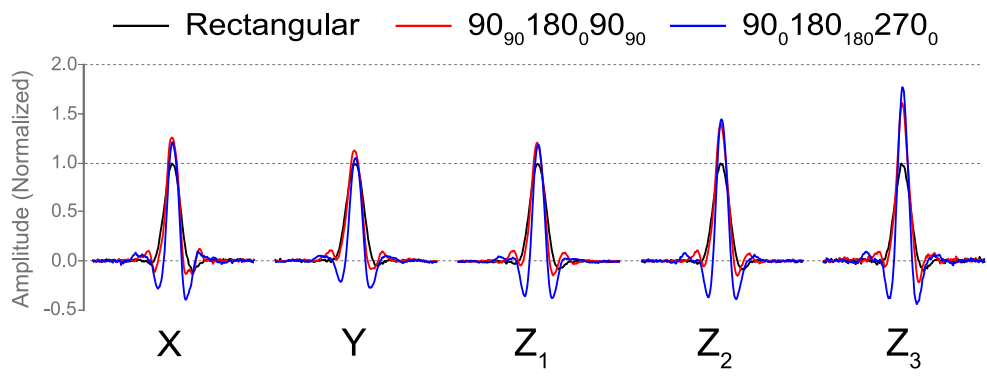

(b)

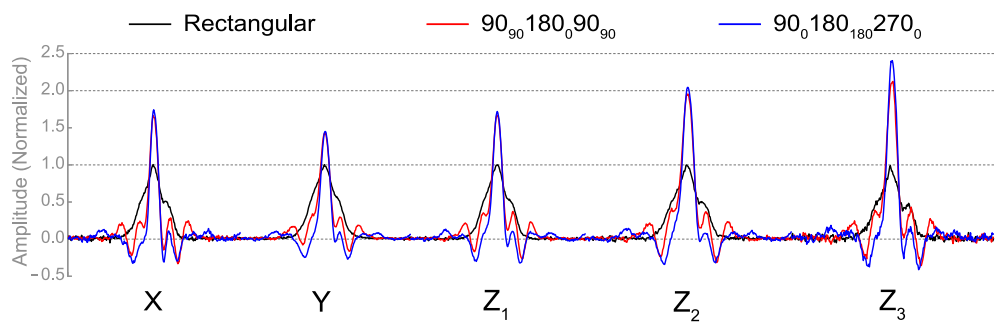

(c)

Figure 11: (a) Absorption spectrum for the nitroxide biradical MSA236, obtained using a field-swept echo experiment, showing positions $\mathrm{X}, \mathrm{Y}, \mathrm{Z}_{1}, \mathrm{Z}_{2}$ and $\mathrm{Z}_{3}$. (b) Refocused echoes obtained using rectangular $16 \mathrm{~ns} \pi$ pulses and composite pulses for corresponding field positions. (c) Repeat of (b) using $32 \mathrm{~ns} \pi$ pulse length. All echoes are normalized to the corresponding rectangular echo. The shoulder to the right of the main peak in the echo is due to $\mathrm{B}_{1}$ inhomogeneity. 
The results show that little enhancement is achieved at positions $\mathrm{X}, \mathrm{Y}$, and $\mathrm{Z}_{1}$, but moderate enhancement is given for $\mathrm{Z}_{2}$ and in particular $\mathrm{Z}_{3}$ where up to 1.78 times echo amplitude is achieved for the $90_{0} 180_{180} 270_{0}$ composite refocused echo. The enhancement is much less than that predicted by the simulation and seen in the neuroglobin Fe(III) experiment (Figure 10).

A quantitative model that fully explains the reduced enhancement is subject to further study, however in this particular model system (where orientations are partially correlated), intra-molecular instantaneous diffusion appears to play a partial role. The phase memory time, $\mathrm{T}_{m}$ is observed to be orientationally dependent, whereas similar measurements on the nitroxyl mono-radical 4-amino-TEMPO, at $50 \mu \mathrm{M}$ concentration, shows no evidence of an orientation dependent phase memory time, and larger enhancements in echo amplitude are observed using composite pulses. The largest enhancement in both cases is seen on $Z_{3}$, and the smallest enhancement on $\mathrm{Y}$ (data shown in Supporting Information).

Larger enhancements are also observed when the excitation bandwidth is reduced. Figure 11(c) shows the results when using 32 ns $\pi$ pulse length. A larger enhancement in echo amplitude is seen for all orientations, with the greatest enhancement for $\mathrm{Z}_{3}$ where the $90_{0} 180_{180} 270_{0}$ composite produced 2.47 times echo amplitude of the rectangular refocused echo. This suggests that good results can be obtained in cases where power is limited.

\subsection{4-pulse DEER for a nitroxide biradical using composite pulses}

DEER experiments were carried out on MSA236 with $90_{90} 180_{0} 90_{90}$ and $90_{0} 180_{180} 270_{0}$ composite pulses used in place of $\pi$ inversion pulses. The combinations of pulse sequences used in this experiment are shown in Figure 
12.

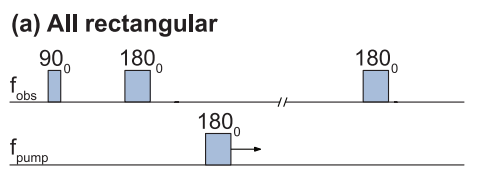

(b) Composite pump

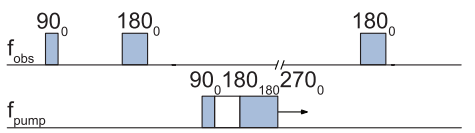

(c) Composite observer

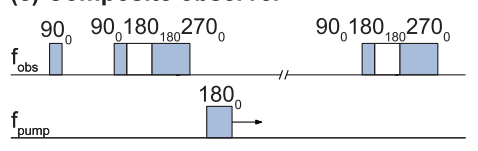

(d) All composite

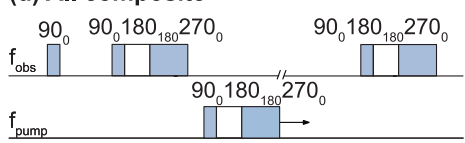

Figure 12: Pulse sequence combinations used in MSA236 composite pulse DEER experiments, shown using the $90_{0} 180_{180} 270_{0}$ composite pulse.

By replacing the pump pulse with a composite (Figure 12(b) composite pump), it is expected that this should increase the modulation depth as the broadband pulse increases the number of inverted partner spins in the dipolar coupling term. The expectation from enhancements seen in the refocused echo experiments, is that by replacing the observer pulse with composite pulses (Figure 12(c) composite observer), it should increase signal to noise with its enhanced echo amplitude. It should be noted that the positions of pump and observer were the same for normal and composite tests. This was to ensure that the same orientations of spins were selected by the pulses.

The DEER experiment was carried out on the YZ orientation (observer $\mathrm{Z}_{3}$ and pump $\mathrm{Y}$ ) using $8 \mathrm{~ns} \pi / 2$ pulse lengths. This orientation was chosen so 
that composite pulses on the pump could be utilized fully to excite the large, broad peak of the spectrum at $Y$. The results for each of the combinations of pulses using the $90_{90} 180_{0} 90_{90}$ and $90_{0} 180_{180} 270_{0}$ composite are shown in Figure 13. The data were processed using DeerAnalysis 2016 [36].
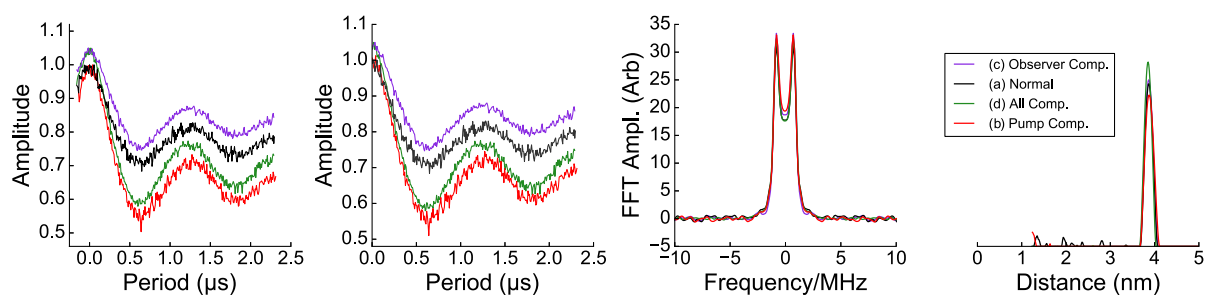

(a)
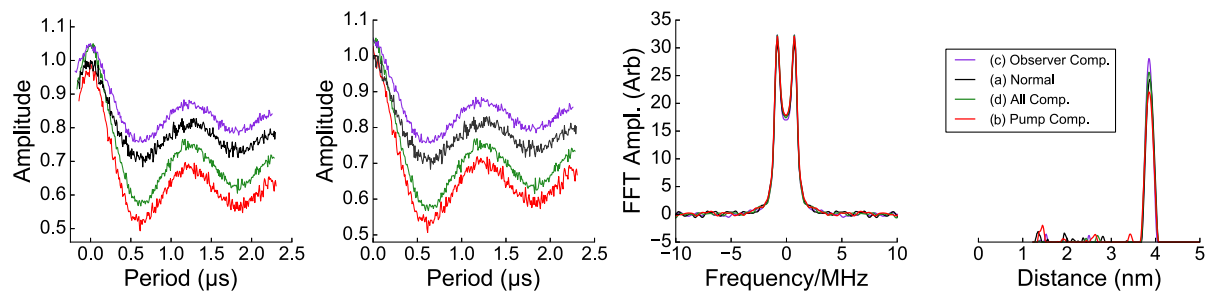

(b)

Figure 13: DEER comparison using (a) $90_{90} 180_{0} 90_{90}$ composite $\pi$ pulse and (b) $90_{0} 180_{180} 270_{0}$ composite $\pi$ pulse. Letters in legend refer to sequences in Figure 12.

The left plots show the raw (normalized) traces, left of center shows the background subtracted data, right of center shows the FFT of the DEER data or Pake pattern, and the right plot shows the derived distance distributions. The observer composite sequence (Figure 12(c)) shown in purple and all $\pi$ composite (Figure 12(d)), shown in green, have been offset vertically by 0.05 for clarity.

Both experiments show no discernible difference between the pump composite and all-composite cases (shown in red and green respectively), which 
each exhibit the same level of modulation enhancement (1.50 times that of the normal, rectangular inversion pulse). The same effect is observed with the rectangular and probe composite sequences (shown in black and purple respectively) showing the same modulation depths also. The signal-to-noise ratio (SNR) does vary between the pairs with the same modulation depth. The SNR for each of the averaged traces was calculated by dividing the magnitude of signal (maximum minus minimum) of the raw DEER data by the standard deviation of the residual noise, which was obtained by subtracting a moving average from the data. The SNR results are summarized in Table 2.

Using this metric, the all- $\pi$ composite $90_{0} 180_{180} 270_{0}$ sequence increases the SNR by 1.76 compared to the rectangular pulse sequence. This is partly due to a decrease in noise, provided by the narrower composite echo and the increase in modulation depth provided by the broadband composite pump. The $90_{90} 180_{0} 90_{90}$ all $\pi$-composite gives 1.62 times enhancement, which is to be expected as it is slightly less broadband than the $90_{0} 180_{180} 270_{0}$ sequence and does not provide as large an echo enhancement. The observer composite and pump composite sequences all provide between 1.27 and 1.38 times enhancement in SNR. When comparing the noise figure and the signal magnitude of the traces to that of the rectangular sequence however, we see that while the pump composite sequences provide the largest absolute increase in modulation depth, the increase in signal level is only 1.45-1.50 times, and the noise level in the trace is observed to slightly increase. In the case of the observer composite sequence however, the signal level or integrated echo area, is similar to that of the rectangular sequence, but the overall SNR improves due 


\begin{tabular}{|l|l|l|l|l|l|}
\hline Pulse Seq. & SNR & $\begin{array}{l}\text { SNR vs. } \\
\text { Rect. }\end{array}$ & $\begin{array}{l}\text { Mod. } \\
\text { Depth vs. } \\
\text { Rect. }\end{array}$ & $\begin{array}{l}\text { Sig. vs. } \\
\text { Rect. }\end{array}$ & $\begin{array}{l}\text { Noise } \\
\text { vs. Rect }\end{array}$ \\
\hline Rectangular & 31.72 & 1.00 & 1.00 & 1.00 & 1.00 \\
\hline Pump $90_{90} 180_{0} 90_{90}$ & 43.72 & 1.38 & 1.57 & 1.45 & 1.05 \\
\hline Pump $90_{0} 180_{180} 270_{0}$ & 40.23 & 1.27 & 1.61 & 1.50 & 1.18 \\
\hline Observer $90_{90} 180_{0} 90_{90}$ & 41.96 & 1.32 & 0.96 & 1.00 & 0.76 \\
\hline Observer $90_{0} 180_{180} 270_{0}$ & 40.99 & 1.29 & 0.94 & 1.03 & 0.79 \\
\hline All $\pi-90_{90} 180_{0} 90_{90}$ & 51.52 & 1.62 & 1.49 & 1.43 & 0.88 \\
\hline All $\pi-90_{0} 180_{180} 270_{0}$ & 55.87 & 1.76 & 1.52 & 1.55 & 0.88 \\
\hline
\end{tabular}

Table 2: SNR comparison of the DEER traces obtained, normalized to the standard rectangular sequence trace

to the reduction of the noise figure by $21-24 \%$, due to the narrower echo. The noise level scales with the number of time samples measured, so by reducing the width of the window, the noise also reduces (c.f. Figure 10(b)). While the echo on the nitroxide does not give the large echo amplitude enhancement as predicted in the simulation and as achieved in the broad low-spin Fe(III) sample, its integrated echo magnitude is the same as the rectangular echo so it does not suffer from a decrease in signal level, but benefits from the decrease in noise. In DEER traces obtained using all $\pi$-composites, the noise decrease is not as large as the observer composite case, but it does benefit from the increase in modulation depth, and these effects combined result in an increase of up to 1.76 times in signal to noise. This equates to a factor of 3 in averaging time. 


\section{Conclusions and outlook}

We have demonstrated that significant gains in echo amplitude, close to the theoretical maximum, can be obtained by replacing standard 16 ns $\pi$ pulses with equivalent composite $\pi$ pulses in the refocused echo detection sequence used in DEER measurements on a broad line low-spin ferric heme system.

However, this enhancement is not as large and varies with orientation when used on a nitroxyl biradical systems although it improves when pulses become more selective. If pulse overlap is minimized, a combined enhancement of up to 1.8 times in SNR using standard pulse lengths has been demonstrated using all-composite $\pi$ pulses in 4-pulse DEER experiments.

In general, it appears to be relatively straightforward to use composite pulses for pump pulses in DEER experiments, where increases in modulation depths by factors of 1.6 were observed using nitroxyl radicals even for the simple and short $90_{90} 180_{0} 90_{90}$ sequence. The main limitation is the extended wings of the excitation, where pulse overlap effects must be evaluated in 4-pulse experiments. However, in cases where power levels are low, and standard pulses are already very selective, composite pulses can provide significant enhancement in modulation depth.

Excitation of coherence is more complicated, and we are not aware of a simple wideband composite $\pi / 2$ pulse sequence that significantly improves over a standard $\pi / 2$ pulse in any type of Hahn echo experiment, which automatically imposes a bandwidth limitation. Gains would also be expected to be limited by the effects of intramolecular, and at higher concentrations, intermolecular instantaneous diffusion. In the Fe(III)-nitroxide neuroglobin 
case instantaneous diffusion is negligible for excitation on the Fe(III). In the nitroxyl biradical system studied, intramolecular effects are believed to play a partial role. However, the echo enhancement with orientation, obtained for the nitroxyl mono-radical (SI) still shows a similar trend in enhancement variability versus orientation, even in the presence of no instantaneous diffusion. This is subject to further study.

In principle, many of these sequences (at least for observer pulses) can also easily be implemented on existing pulse EPR instruments with phase cycling capability and are likely to be effective in cases where power is limited. Up to 2.4 times echo enhancement for broad line systems is expected using standard resonators using $90_{0} 180_{180} 270_{0}$ composite pulses.

However, the recent introduction of Arbitrary Waveform Generators into commercial spectrometers has opened the possibility of increased sensitivity by very wideband excitation using chirped pulses. These gains can be limited by cavity bandwidth but in this paper, we have demonstrated a high frequency system with sufficient bandwidth to incorporate fast phase changes required for wideband composite pulse sequences, without any need for compensation or making any compromises in the overall sensitivity of the instrument. This suggests that there is still significant scope to further improve sensitivity for broadline systems before being limited by the available bandwidth.

We have demonstrated that composite pulses can represent a practical solution to improving sensitivity particularly when the length of the pulse sequence is limited. The bandwidth advantages of non-resonant systems operating in induction mode are equally applicable to any optimal control 
sequence.

\section{Acknowledgments}

C.L.M. acknowledges funding from EPSRC as part of the iMRCDT.

The W-band instrument was developed under the U.K. Research Councils Basic Technology Program (grant EP/F039034/1).

S.V.D. acknowledges the Research Foundation Flanders (FWO) for financial support (grant G.0687.13).

J.E.L. thanks the Royal Society for a University Research Fellowship.

Sylvia Dewilde (Biomedical Sciences, University of Antwerp) is thanked for the purification of the Cys46Ser/ Cys55Ser mutant of human neuroglobin (NGB) used in this work.

Adelheid Godt's group is thanked for the synthesis of MSA236.

We also thank the Wellcome Trust (grant 099149/Z/12/Z).

\section{Appendix A. Data availability}

The research data (and/or materials) supporting this publication can be accessed at http://dx.doi.org/10.17630/b65d05e6-6efa-48b9-a741-5a6322159a4a

[1] M. H. Levitt, R. Freeman, NMR population inversion using a composite pulse, Journal of Magnetic Resonance 33 (2) (1979) 473-476. doi:10.1016/0022-2364(79)90265-8.

[2] K. Uurbil, M. Garwood, M. Robin Bendall, Amplitude- and frequencymodulated pulses to achieve $90^{\circ}$ plane rotations with inhomogeneous B1 fields, Journal of Magnetic Resonance 72 (1) (1987) 177-185. doi:10.1016/0022-2364(87)90186-7. 
[3] P. E. Spindler, S. J. Glaser, T. E. Skinner, T. F. Prisner, Broadband inversion PELDOR spectroscopy with partially adiabatic shaped pulses., Angewandte Chemie (International ed. in English) 52 (12) (2013) 34259. doi:10.1002/anie.201207777.

[4] N. Khaneja, R. Brockett, S.J. Glaser, Time optimal control in spin systems, Phys. Rev. A 63 (2001) 32308. doi:10.1103/PhysRevA.63.032308.

[5] A. Doll, S. Pribitzer, R. Tschaggelar, G. Jeschke, Adiabatic and fast passage ultra-wideband inversion in pulsed EPR, Journal of Magnetic Resonance 230 (2013) 27-39.

[6] C. L. Motion, J. E. Lovett, S. Bell, S. L. Cassidy, P. A. S. Cruickshank, D. R. Bolton, R. I. Hunter, H. El Mkami, S. Van Doorslaer, G. M. Smith, DEER Sensitivity between Iron Centers and Nitroxides in Heme-Containing Proteins Improves Dramatically Using Broadband, High-Field EPR, The Journal of Physical Chemistry Letters 7 (8) (2016) 1411-1415. doi:10.1021/acs.jpclett.6b00456.

[7] R. H. Crepeau, A. Dulć, J. Gorcester, T. R. Saarinen, J. H. Freed, Composite pulses in time-domain ESR, Journal of Magnetic Resonance (1969) 84 (1) (1989) 184-190. doi:10.1016/0022-2364(89)90017-6.

[8] J. Morton, A. Tyryshkin, A. Ardavan, K. Porfyrakis, S. Lyon, G. Briggs, High Fidelity Single Qubit Operations Using Pulsed Electron Paramagnetic Resonance, Physical Review Letters 95 (20) (2005) 200501. doi:10.1103/PhysRevLett.95.200501. 
[9] I. V. Koptyug, S. H. Bossmann, N. J. Turro, Inversion-Recovery of Nitroxide Spin Labels in Solution and Microheterogeneous Environments, Journal of the American Chemical Society 118 (6) (1996) 1435-1445. doi:10.1021/ja952152d.

[10] M. Pannier, S. Veit, A. Godt, G. Jeschke, H. Spiess, DeadTime Free Measurement of DipoleDipole Interactions between Electron Spins, Journal of Magnetic Resonance 142 (2) (2000) 331-340. doi:10.1006/jmre.1999.1944.

[11] A. D. Milov, A. B. Ponomarev, Y. Tsvetkov, Electron-electron double resonance in electron spin echo: Model biradical systems and the sensitized photolysis of decalin, Chemical Physics Letters 110 (1) (1984) 67-72. doi:10.1016/0009-2614(84)80148-7.

[12] G. W. Reginsson, O. Schiemann, Pulsed electron-electron double resonance: beyond nanometre distance measurements on biomacromolecules., The Biochemical journal 434 (3) (2011) 353-63. doi:10.1042/BJ20101871.

[13] O. Schiemann, T. F. Prisner, Long-range distance determinations in biomacromolecules by EPR spectroscopy., Quarterly reviews of biophysics 40 (1) (2007) 1-53. doi:10.1017/S003358350700460X.

[14] J. E. Banham, C. M. Baker, S. Ceola, I. J. Day, G. H. Grant, E. J. J. Groenen, C. T. Rodgers, G. Jeschke, C. R. Timmel, Distance measurements in the borderline region of applicability of CW EPR and DEER: a model study on a homologous series of spin-labelled peptides., Journal 
of magnetic resonance (San Diego, Calif. : 1997) 191 (2) (2008) 202-18. doi:10.1016/j.jmr.2007.11.023.

[15] D. Goldfarb, Gd(3+) spin labeling for distance measurements by pulse EPR spectroscopy., Physical chemistry chemical physics : PCCP 16 (21) (2014) 9685-99. doi:10.1039/c3cp53822b.

[16] A. M. Raitsimring, C. Gunanathan, A. Potapov, I. Efremenko, J. M. L. Martin, D. Milstein, D. Goldfarb, Gd3+ complexes as potential spin labels for high field pulsed EPR distance measurements., Journal of the American Chemical Society 129 (46) (2007) 14138-9. doi:10.1021/ja075544g.

[17] A. M. Bowen, M. W. Jones, J. E. Lovett, T. G. Gaule, M. J. McPherson, J. R. Dilworth, C. R. Timmel, J. R. Harmer, Exploiting orientationselective DEER: determining molecular structure in systems containing $\mathrm{Cu}$ (II) centres, Phys. Chem. Chem. Phys. 18 (Ii) (2016) 5981-5994. doi:10.1039/C5CP06096F.

[18] A. Doll, M. Qi, N. Wili, S. Pribitzer, A. Godt, G. Jeschke, Gd(III)Gd(III) distance measurements with chirp pump pulses., Journal of magnetic resonance (San Diego, Calif. : 1997) 259 (2015) 153-62. doi:10.1016/j.jmr.2015.08.010.

[19] G. Jeschke, M. Pannier, A. Godt, H. Spiess, Dipolar spectroscopy and spin alignment in electron paramagnetic resonance, Chemical Physics Letters 331 (2-4) (2000) 243-252. doi:10.1016/S0009-2614(00)01171-4. 
[20] P. Schöps, P. E. Spindler, A. Marko, T. F. Prisner, Broadband spin echoes and broadband SIFTER in EPR., Journal of magnetic resonance (San Diego, Calif. : 1997) 250 (2015) 55-62. doi:10.1016/j.jmr.2014.10.017.

[21] A. Doll, G. Jeschke, EPR-correlated dipolar spectroscopy by Q-band chirp SIFTER, Physical Chemistry Chemical Physics 18 (33) (2016) 23111-23120. doi:10.1039/b000000x.

[22] M. H. Levitt, Composite Pulses, in: eMagRes, Wiley, Chichester, UK, 2007, pp. 1396-1411. doi:10.1002/9780470034590.emrstm0086.

[23] A. Shaka, R. Freeman, A composite 180 pulse for spatial localization, Journal of Magnetic Resonance (1969) 63 (3) (1985) 596-600. doi:10.1016/0022-2364(85)90252-5.

[24] M. H. Levitt, Symmetrical composite pulse sequences for NMR population inversion. I. Compensation of radiofrequency field inhomogeneity, Journal of Magnetic Resonance 48 (2) (1982) 234-264. doi:10.1016/00222364(82)90275-X.

[25] R. Tycko, a. Pines, J. Guckenheimer, Fixed point theory of iterative excitation schemes in NMR, The Journal of Chemical Physics 83 (6) (1985) 2775. doi:10.1063/1.449228.

[26] S. Odedra, S. Wimperis, Use of composite refocusing pulses to form spin echoes, Journal of Magnetic Resonance 214 (1) (2011) 68-75. doi:10.1016/j.jmr.2011.10.006. 
[27] A. Shaka, A. Pines, Symmetric phase-alternating composite pulses, Journal of Magnetic Resonance (1969) 71 (3) (1987) 495-503.

[28] P. A. S. Cruickshank, D. R. Bolton, D. A. Robertson, R. I. Hunter, R. J. Wylde, G. M. Smith, A kilowatt pulsed 94 GHz electron paramagnetic resonance spectrometer with high concentration sensitivity, high instantaneous bandwidth, and low dead time, Review of Scientific Instruments 80 (10) (2009) 103102. doi:10.1063/1.3239402.

[29] G. M. Smith, P. A. S. Cruickshank, D. R. Bolton, D. A. Robertson, Highfield pulse EPR instrumentation, in: Electron Paramagnetic Resonance: Volume 21, Vol. 21, The Royal Society of Chemistry, Cambridge, UK, 2008, pp. 216-233. doi:10.1039/B807958G.

[30] S. Bhat, A. Sharma, S. Bhat, Vitrification and Glass Transition of Water: Insights from Spin Probe ESR, Physical Review Letters 95 (23) (2005) 235702. doi:10.1103/PhysRevLett.95.235702.

[31] M. Ezhevskaya, E. Bordignon, Y. Polyhach, L. Moens, S. Dewilde, G. Jeschke, S. Van Doorslaer, Distance determination between lowspin ferric haem and nitroxide spin label using DEER: the neuroglobin case, Molecular Physics 111 (18-19) (2013) 2855-2864. doi:10.1080/00268976.2013.813592.

[32] G. Jeschke, M. Sajid, M. Schulte, N. Ramezanian, A. Volkov, H. Zimmermann, A. Godt, Flexibility of shape-persistent molecular building blocks composed of p-phenylene and ethynylene units, Jour- 
nal of the American Chemical Society 132 (29) (2010) 10107-10117. arXiv:arXiv:1011.1669v3, doi:10.1021/ja102983b.

[33] A. D. Olver, P. J. B. Clarricoats, A. A. Kishk, L. Shafai, Microwave Horns and Feeds, IET, The Institution of Engineering and Technology, Michael Faraday House, Six Hills Way, Stevenage SG1 2AY, UK, 1994. doi:10.1049/PBEW039E.

[34] P. J. Clarricoats, A. Olver, Corrugated horns for microwave antennas, Electromagnetic Waves, Institution of Engineering and Technology, 1984. doi:10.1049/PBEW018E.

[35] E. A. Nanni, A. B. Barnes, Y. Matsuki, P. P. Woskov, B. Corzilius, R. G. Griffin, R. J. Temkin, Optimization of $\mathrm{THz}$ wave coupling into samples in DNP/NMR spectroscopy, in: 35th International Conference on Infrared, Millimeter, and Terahertz Waves, IEEE, 2010, pp. 1-3. doi:10.1109/ICIMW.2010.5612956.

[36] G. Jeschke, V. Chechik, P. Ionita, A. Godt, H. Zimmermann, J. Banham, C. R. Timmel, D. Hilger, H. Jung, DeerAnalysis2006a comprehensive software package for analyzing pulsed ELDOR data, Applied Magnetic Resonance 30 (3-4) (2006) 473-498. doi:10.1007/BF03166213. 